Handlungsoptionen für eine Integration von Arbeitsmarkt- und Verkehrspolitik

\section{Zwei Politikwelten?}

\section{Der wirtschaftliche Strukturwandel und die Trends zur Flexibilisierung der Arbeitswelt sind mit tief greifenden Wirkungen auf die Mobilitätsnachfrage und -organisation verbunden. Diese materialisieren sich im Verkehr. Planungs- verfahren und Politik, die auf traditionellen "starren" Beziehungsmustern aufbauen und auf den Verkehrsbereich begrenzt bleiben, werden dadurch zunehmend in Frage gestellt. Neue Lösungswege müssen gesucht werden (1).}

$\mathrm{D}$ Von Heike Flämig und Ingo Einacker e Organisation der Arbeitswelt hat erhebliche Auswirkungen auf das Verkehrssystem. Zu den überwiegend beeinflussbaren Größen gehören die Ausgestaltung von Arbeit in Zeit, Inhalt, Organisation und Erwerbsform sowie die berufliche Qualifikation.

Hinsichtlich der Mobilitätswirkungen spielt vor allem der Wandel von Erwerbsformen durch zeitpolitische Entwicklungslinien eine wichtige Rolle. Erstens wird die Arbeitszeit kürzer und flexibler. Zweitens tritt eine Pluralisierung der Arbeitszeitmuster auf: Das traditionelle Normalarbeitsverhältnis geht weiter zurück, flexible Arbeitszeitmodelle, Teilzeitarbeit und selbständige Erwerbsformen gewinnen an Bedeutung. Drittens haben die Zeitarbeit und - bis zur Neuregelung im April 1999 - die Anzahl der Scheinselbständigen und geringfügig Beschäftigten stark zugenommen.

Neben den Arbeitszeitstrukturen hängt das Mobilitätsverhalten stark von der Bereitschaft der Menschen ab, weite Arbeitswege in Kauf zu nehmen, um einen Arbeitsplatz zu erhalten oder zu sichern. Da die Bindung an den Wohnort meist langfristiger ist als die Bindung an einen Arbeitsplatz, nehmen die zurückgelegten Entfernungen im Berufsverkehr zu.

\section{Ambivalente Wirkungen}

Gleichzeitig sind die Veränderung der Arbeitswelt in ihrer Wirkung auf den Verkehrsbereich ambivalent zu beurteilen, wie die folgenden Beispiele illustrieren:

- Eine steigende Flexibilisierung der täglichen bzw. wöchentlichen Arbeitszeit senkt die Spitzennachfrage im Berufsverkehr und bildet individuelle Mobilitätsbedürfnisse aus. Durch die Auflösung kollektiver Zeitmuster wird die Nutzung des ÖPNV und die Bildung von Fahrgemeinschaften erschwert und im heutigen Verkehrssystem der Pkw begünstigt.
Die zunehmende Frauenerwerbstätigkeit und die vielfach anzutreffenden Teilzeitarbeitsplätze sowie die Doppelbelastung der Frauen (Koordinierung Haushalt-Familie-Arbeit) tragen zu einer erhöhten Mobilitätsnachfrage und veränderten Mobilitätsorganisation bei, die noch verstärkt wird, wenn mehreren (geringfiigigen) Beschäftigungsverhältnissen nachgegangen wird.

- Die inhaltliche Veränderung der Arbeitswelt (Dienstleistungsgesellschaft) führt zu veränderten räumlichen und zeitlichen Arbeitsrhythmen.

- Höher Qualifizierte sind in der Regel mobiler - sowohl im täglichen Mobilitätsverhalten als auch bezogen auf Betriebswechsel.

- Durch neue Informations- und Kommunikationstechniken entstehen neue Formen der Beschättigung und Arbeitsorganisation (Telearbeit, Telekonferenzen usw.). Diese wirken in unterschiedlicher Richtung auf das Mobilitätssystem. Einerseits können sie teilweise physischen Verkehr substituieren. Andererseits verändert sich beispielsweise bei der weit verbreiteten alternierenden Telearbeit das Mobilitätsverhalten, was in der Regel die Mobilitätsnachfrage und die Flexibilitätsanforderungen an das Verkehrssystems erhöht.

Hier gibt es darüber hinaus einen weiteren $\mathrm{Zu}$ sammenhang mit der Branchenstruktur bzw. mit Berufen, in denen laut Pendlerstatistik stark ausdifferenzierte Mobilitätsstile vorzufinden sind. Im Durchschnitt aller Berufsgruppen pendelten 1998 in Nordrhein-Westfalen rund 48 Prozent aller am Arbeitsort beschäftigten Personen. Die höchste Einpendlerquote wurde dabei mit fast 70 Prozent für die Berufsgruppe der Ingenieure, Chemiker, Physiker und Mathematiker festgestellt. Hingegen pendeln nur rund ein Drittel derjenigen, die in Reinigungs-, Körperpflege- und Hauswirtschaftsberufen tätig sind. Eine detailliertere Analyse der Pendlerverflechtungen von vier nordrhein-westfälischen Städten hat außerdem gezeigt, dass es einen deutlichen Zusammenhang von Mobilitätsnachfrage und der Einwohnerzahl einer Stadt, ihrer zentralörtlichen Bedeutung einschließlich ihrer Standortattraktivität und Beschäftigungsprognose gibt. Neben veränderten Arbeitszeiten, -inhalten und -organisationen spielen demnach auch Faktoren außerhalb der eigentlichen Erwerbsarbeit eine bedeutende Rolle für Mobilitätsnachfrage und -organisation.

\section{- Handlungsoptionen}

Inzwischen gibt es eine Vielzahl an Beispielsammlungen für mögliche Maßnahmen, Instrumente und Ansätze im Handlungsfeld Verkehr und Mobilität (2). Diese beziehen sich schwerpunktmäßig auf die Verbesserung des Verkehrssystems selbst, wie die qualitative Verbesserung im Umweltverbund, die Verbesserung der Güterverkehrsinfrastruktur und die Förderung des Mobilitätsmanagements.

Die größten Potenziale zur Realisierung von positiven Beschäftigungs- oder Verkehrseffekten liegen unseres Erachtens im Bereich derjenigen Ansätze, die eine an der Reduzierung bzw. Eindämmung des Verkehrswachstums orientierte Verkehrs- und Mobilitätspolitik in andere Politikfelder hineintragen. Dazu gehören vor allem strukturelle Ansätze, die die auf Raum, Zeit und Bauten Einfluss nehmen, sowie organisatorische Ansätze, die zum Beispiel zur Stärkung regionaler Wirtschaftskreisläufe beitragen. Anknüpfend an den Ergebnissen der Wirkungsanalyse zum Problembereich Arbeit und Mobilität (1) handelt es sich einerseits um Handlungsoptionen zur Beeinflussung der Ansiedlung von Haushalten und Unternehmen und anderseits um Ansätze im Bereich der Arbeitsmarktpolitik.

\section{Standortwahl beeinflussen}

Mögliche Instrumente zur Reduzierung von Distanzen zwischen Wohn- und Arbeitsort sind eine von der Berufsverkehrsdistanz abhängige Eigenheimförderung und eine vom Gesamtverkehrsaufwand abhängige regionale Wirtschaftsförderung (3). Diese Instrumente müssen durch ein entsprechendes Quartiersmanagement flankiert werden: durch ein verkehrssparsames Belegungsmanagement für Wohnungen und durch eine verkehrssparsame Ansiedlungspolitik für Unternehmen. Gefordert sind daher

- die Forcierung einer innovationsorientierten Bestandspolitik, schwerpunktmäßig bezogen auf lokale und regionale Netzwerke kleinerer und mittlerer Unternehmen. 
die Überarbeitung bzw. Ergänzung der Fördergrundsätze und -voraussetzungen der regionalen Wirtschaftsförderung um verkehrliche Kriterien.

- eine frühzeitige Berücksichtigung aller Verkehre (Mitarbeiter, Kunden, Besucher, Lieferanten, Entsorger) bei der Gewerbestandortplanung, beispielsweise durch eine rechtzeitige Verzahnung mit einem Mobilitätsmanagement oder durch ABC-Planung.

Dafür ist es notwendig, dass die Reduzierung negativer verkehrlicher Wirkungen als Folge von Entscheidungen, Regelwerken und Maßnahmen auf allen politischen Ebenen erklärter politischer Wille wird. Ein Instrument ist die vom Verkehrsauswirkungsprüfung, die seit 1996 als vom Bundesverkehrsministerium herausgegebene „Prüffragen zur Verkehrsauswirkung von Gesetzes und Verordnungsvorhaben des Bundes“ vorliegt.

\section{- Arbeitsmarktpolitik}

Die allgemeine Entwicklung am Arbeitsmarkt, die Ausgestaltung von Erwerbstätigkeit und die Flexibilisierung von Arbeitszeitsystemen hat eine hohe Bedeutung für die Mobilitätsnachfrage und die Mobilitätsorganisation. Der Arbeitsmarktpolitik kommt somit eine wichtige Rolle bei der Mobilitätsgestaltung $\mathrm{zu}$, die sie durch verschiedene Handlungsstränge füllen kann:

Regionalisierte Arbeitsmarktpolitik: Durch eine wesentlich verstärkte regionale und lokale Ausrichtung der arbeitsmarktpolitischen Maßnahmen kann es gelingen, von Arbeitslosigkeit bedrohten Arbeitnehmern und arbeitlosen Personen eine Beschäftigung in der eigenen Region zu sichern bzw. zu schaffen, um so unerwünschte Effekte auf die Mobilität durch Pendlerströme zu vermeiden.

Integrierte Arbeitsmarktpolitik: Die Wirksamkeit arbeitsmarktpolitischer Maßnahmen kann durch eine enge Verzahnung der Arbeitsmarktpolitik mit Maßnahmen und Konzepten aus anderen Politikfeldern erhöht werden, beispielsweise können durch Nutzungsvorbereitung und Vermarktung ehemaliger Industrieflächen Synergieeffekte im Sinne einer nachhaltigen Aufwertung des Wirtschaftsstandortes genutzt werden, mit positiven Rahmensetzungen für die Mobilitätsnachfrage.

Förderung neuer Arbeitszeitmodelle: Die Wirkungsanalyse hat deutlich gemacht, dass neue Arbeitszeitmodelle unterschiedliche Implikationen für die Mobilität besitzen. Diese teilweise sehr deutlichen Auswirkungen auf den Verkehrssektor müssen im Rahmen politischer Maßnah- men zur Flexibilisierung der Arbeitszeit mitgedacht werden. Sie sollten in Tarifverträgen und Vereinbarungen berücksichtigt werden.

Förderung neuer Mobilitätsdienstleistungen: Vor dem Hintergrund sich wandelnder Rahmenbedingungen scheint die Durchsetzung moderner Dienstleistungen im Verkehr ein vielversprechender Ansatz zur umweltfreundlichen Abwicklung des beschäftigungsbedingten Verkehrs und zur intermodalen Verknüpfung der verschiedenen Verkehrsträger zu sein. Die Einführung, Förderung und Stärkung des Mobilitätsmanagements sollte nicht nur Aufgabe von Arbeitsmarkt- und Verkehrspolitik des Landes sein, sondern vor allem auch der Betriebspolitik.

\section{Schlussfolgerungen}

Arbeitsmarkt- und Verkehrspolitik sind beide abgeleitete Größen. Viele Bestimmungsgrößen, die Einfluss auf die Entwicklung der Arbeitswelt und der Mobilitätsnachfrage und -organisation nehmen, sind Ergebnisse komplexer Wirkungsgebilde. Daher ist eine treffsichere Vorhersage einer zukünftigen Ausgestaltung der Arbeitswelt und von Mobilität kaum möglich.

Die Begrenztheit der direkten Steuerungspotenziale von Verkehrswachstum und Verkehrserzeugung geht nicht nur auf direkte verkehrssysteminterne Zusammenhänge von Verkehrsinfrastruktur, Verkehrsangeboten, Verkehrskosten und daraus resultierenden Verkehrsverhalten zurück. Sie resultiert vor allem aus externen, indirekten Wachstumsimpulsen und Wirkungszusammenhängen, vor allem im Bereich der Arbeit aber auch im Bereich von Städtebau und Umweltplanung sowie der Wirtschaftsförderung und der Ansiedlungspolitik, insbesondere der räumlichen Planung. Gesucht sind daher auch Gestaltungsmöglichkeiten außerhalb des Verkehrssystems, im so genannten Verkehrsumfeld (4).

Verkehrspolitik wirkt in andere Politikbereiche ein und muss dies auch tun, will sie etwas bewegen. Dies bedeutet aber auch, dass die Ziele der Verkehrspolitik mit den Zielen anderer Politikbereiche in Einklang gebracht werden müssen, und dies möglichst auf allen Ebenen. Mobilitätspolitik muss daher als Querschnittspolitik und -planung verstanden werden. Dies gilt nicht nur für eine aktive Beeinflussungsplanung von Siedlungs- und Standortentwicklung. Dies würde eine einseitige Konzentration auf baulich-räumliche Rahmenbedingungen bedeuten. Vielmehr besteht die Notwendigkeit, bereits am Ort der Entscheidung anzusetzen, also dort, wo über eine räum- lich-zeitliche Arbeitsteilung entschieden wird, die die Siedlungsentwicklung beeinflusst und die Mobilitätsnachfrage und -organisation nachhaltig bestimmt.

\section{Anmerkungen}

(1) Der Beitrag beruht auf Ergebnissen einer Studie des IÖW für die Enquête-Kommission „Zukunft der Mobilität” des Landtags Nordrhein-Westfalen. Vgl. Flämig, Heike/ Einacker, Ingo/ Petschow, Ulrich/Schumacher, Günter: Arbeit und Mobilität - zukünftige Handlungsoptionen in NordrheinWestfalen. Die Zukunft der Arbeit als Ausgangspunkt für Veränderungen in der Mobilitätsnachfrage und der Mobilitätsorganisation. IÖW-Schriftenreihe 153/00, Berlin 2000. (2) Vgl. z.B. Hans-Böckler-Stiftung/ Deutscher Gewerkschaftsbund (Hrsg.): Strategien für die Mobilität der Zukunft. Handlungskonzepte für lokale, regionale und betriebliche Akteure, Düsseldorf 2001 und Verkehrsclub Deutschland (Hrsg.): Mobilitätsmanagement in Betrieb und Verwaltung. Bonn 1996. Eine aktuelle Sammlung von Kurzbeispielen des Deutschen Instituts für Urbanistik findet sich im Internet unter http://www.difu.de/stadtoekologie/praxis (Stichwort Mobilität/Verkehr).

(3) Die Grundlage bilden die Ergebnisse eines Forschungsvorhabens des Umweltbundesamtes, das gemeinsam vom Büro für Integrierte Planung (BIP) und dem IÖW durchgeführt wurde. Vgl. UBA (Hrsg.): Quantifizierung der Verkehrsentstehung und deren Umweltauswirkungen durch Entscheidungen, Regelwerke und Maßnahmen mit indirektem Verkehrsbezug. UBA-Texte 35/00, Berlin 2000.

(4) Vgl. auch die Beiträge des Schwerpunkts "Städtische Nutzung und Verkehr" der Ausgabe 5-6/99 von Ökologisches Wirtschaften.

\section{Die Autorlnnen}

Heike Flämig ist wissenschaftliche Mitarbeiterin an der Technischen Universität Hamburg-Harburg. Kontakt: European Centre for Transportation and Logistics (ECTL), TU Hamburg-Harburg Arbeitsbereich Verkehrssysteme und Logistik, AB 1-10, 21071 Hamburg, Tel. 040/ 42878-3907, Fax -2728, E-mail: flaemig@tu-harburg.de Ingo Einacker ist wissenschaftlicher Mitarbeiter im Forschungsfeld Stadtentwicklung, Planung, Verkehr des Instituts für ökologische Wirtschaftsforschung. Kontakt: IÖW, Potsdamer Str. 105, 10785 Berlin. Tel. 030/ 884594-19, Fax 030/ 8825439, E-mail: ingo.einacker@ioew.de 
(c) 20I0 Authors; licensee IÖW and oekom verlag. This is an article distributed under the terms of the Creative Commons Attribution Non-Commercial No Derivates License (http://creativecommons.org/licenses/by-nc-nd/3.o/), which permits unrestricted use, distribution, and reproduction in any medium, provided the original work is properly cited. 